FACTA UNIVERSITATIS

Series: Mechanical Engineering Vol. 18, N 3, 2020, pp. 419 - 437

https://doi.org/10.22190/FUME200602034P

Original scientific paper

\title{
PRIORITIZING THE WEIGHTS OF THE EVALUATION CRITERIA UNDER FUZZINESS: THE FUZZY FULL CONSISTENCY METHOD - FUCOM-F
}

\author{
Dragan Pamucar ${ }^{1}$, Fatih Ecer $^{2}$ \\ ${ }^{1}$ Department of Logistics, University of Defence in Belgrade, Military Academy, \\ Belgrade, Serbia \\ ${ }^{2}$ Department of Business Administrative, Faculty of Economics and Administrative \\ Sciences, Afyon Kocatepe University, Afyonkarahisar, Turkey
}

\begin{abstract}
Values, opinions, perceptions, and experiences are the forces that drive almost each and every kind of decision-making. Evaluation criteria are considered as sources of information used to compare alternatives and, as a result, make selection easier. Seeing their direct effect on the solution, weighting methods that most accurately determine criteria weights are needed. Unfortunately, the crisp values are insufficient to model real life problems due to the lack of complete information and the vagueness arising from linguistic assessments of decision-makers. Therefore, this paper proposes a novel subjective weighting method called the Fuzzy Full Consistency Method (FUCOM-F) for determining weights as accurately as possible under fuzziness. The most prominent feature of the proposed method is obtaining the most accurate weight values with very few pairwise comparisons. Consequently, thanks to this model, consistency and reliability of the results increase while the processing time and effort decrease. Moreover, an illustrative example related to the green supplier evaluation problem is performed. Finally, the robustness and effectiveness of the proposed fuzzy model is demonstrated by comparing it with fuzzy best-worst method ( F-BWM) and fuzzy AHP (F-AHP) models.
\end{abstract}

Key Words: Fuzzy Full Consistency Method (FUCOM-F), Weighting of Criteria, MCDM, Subjective Weighting, Fuzzy BWM, Fuzzy AHP

\footnotetext{
Received June 02, 2020 / Accepted August 18, 2020

Corresponding author: Fatih Ecer

Department of Business Administrative, Faculty of Economics and Administrative Sciences, Afyon Kocatepe

University, ANS Campus, 03030 Afyonkarahisar, Turkey

E-mail: fatihecer@gmail.com

C 2020 by University of Niš, Serbia | Creative Commons License: CC BY-NC-ND
} 


\section{INTRODUCTION}

Multi-criteria decision-making (MCDM), which is a very important component of the decision-making theory, is usually divided into two classes with regard to the solution area of the problem, as continuous and discrete. In order to address continuous problems, multi-objective decision-making (MODM) methods are adopted. However, discrete problems are solved by using multi-attribute decision-making (MADM) methods. Nonetheless, MCDM is widely used to describe the discrete MCDM i.e. MADM in existing literature [1].

The MCDM methods aim at selecting the best alternative among those available. One of the two main components of the MCDM methods is represented by the weights of the criteria that define the process under consideration. The weights of the criteria express the importance and the effects of the criteria on the evaluation results. Criterion weights can be determined subjectively or objectively. Moreover, opinions, thoughts, and experiences of experts play a crucial role whilst criterion weights are subjectively determined [2].

As mentioned above, determining the weights of the criteria is one of the key problems that arise in multi-criteria analysis models. The problem of selecting an appropriate method for defining the weight coefficients of criteria is a very important step in the models of MCDM. The impartial determination of weight coefficients and the transformation of stated expert preferences into weight coefficients are basic requirements that are posed before the subjective group of models. If we bear in mind that the weight coefficients significantly influence the outcome of a decision-making process, it is clear that particular attention has to be paid to the models for determining the weights of criteria.

Numerous authors $[3,4,5]$ agree that the values of the criteria weights are significantly conditioned by the methods of their determination. In addition, there is no agreement on the best method of determining criteria weights. However, in the literature there is an agreement that the weights calculated by certain methods are significantly more accurate than those obtained by expert evaluations. In their study, Zavadskas et al. [6] found that the Analytic Hierarchy Process (AHP) is the most commonly used model for determining the weight coefficients of criteria and/or the evaluation of alternatives. One of the benefits and reasons as to why the authors opt for the application of the AHP model is due to the ability to validate results by determining the degree of the model consistency. However, according to some psychological research [7], in the AHP method it is very difficult to perform completely consistent pairwise comparisons over nine criteria since this requires a large number of comparisons $(\mathrm{n}(\mathrm{n}-1) / 2)$.

A model that has managed to overcome some of the above-mentioned AHP model constraints is the Best-Worst Method (BWM) [1]. One of the greatest advantages of the BWM is a significantly lower number of pairwise comparisons compared to the AHP, only 2 n-3. A smaller number of pairwise comparisons of criteria have a direct impact on higher consistency of the model, i.e. greater reliability of the results. Additionally, the application of the BWM is not limited to comparing up to nine criteria as it requires a lower number of comparisons. By forming Best-to-Others (BO) and Others-to-Worst (OW) vectors, the data that are more consistent are obtained through the AHP model with a lower number of pairwise comparisons, at the same time. However, one of the problems with the BWM is determination of the optimum values of weight coefficients in the case of major deviations in the degree of consistency. In such situations, Rezaei [1] proposes 
the determination of interval values and use of the mean value of intervals as the final value of the weight coefficient. However, there is no guarantee that the central part of the interval will represent the optimal weight coefficients values. The optimum value may be closer to the left or right limit of the interval. In the cases of greater inconsistency of results, the optimum values of weight coefficients are not even covered by the defined interval weight values [8].

One of the newer models for determining the criteria weights, based on the principles of the pairwise comparisons of criteria and the validation of results throughout a deviation from maximum consistency, is the Full Consistency Method (FUCOM) [9]. The FUCOM is a model that, to some extent, eliminates the stated deficiencies of the BWM and AHP models. As shown in Fig. 1, the advantages that are determinative for the application of the FUCOM include a small number of pairwise comparisons of criteria (only $n-1$ comparison), the ability to validate the results by defining the deviations from the maximum consistency (DMC) of comparisons, and appreciation of transitivity during the pairwise comparison of criteria. As with other subjective models for determining the weights of criteria, in the FUCOM model there is a subjective influence of decisionmakers on the final values of the weights of criteria. This particularly refers to the first and second step of the FUCOM in which decision-makers rank the criteria according to their personal preferences and perform pairwise comparisons of the criteria ranked. However, unlike other subjective models, the FUCOM has shown minor deviations in the obtained values of the weights of criteria from the optimum values [9]. Moreover, the methodological procedure of the FUCOM eliminates the problem of redundancy of pairwise comparisons of criteria, which is present in some subjective models for determining the weights of criteria $[10,11,12]$.

In addition to being a new model, there are a number of studies in which the benefits of the FUCOM are exploited. For example, Pamucar et al. [8] demonstrated the application of the FUCOM-MAIRCA multi-criteria model for evaluating the railway crossings.

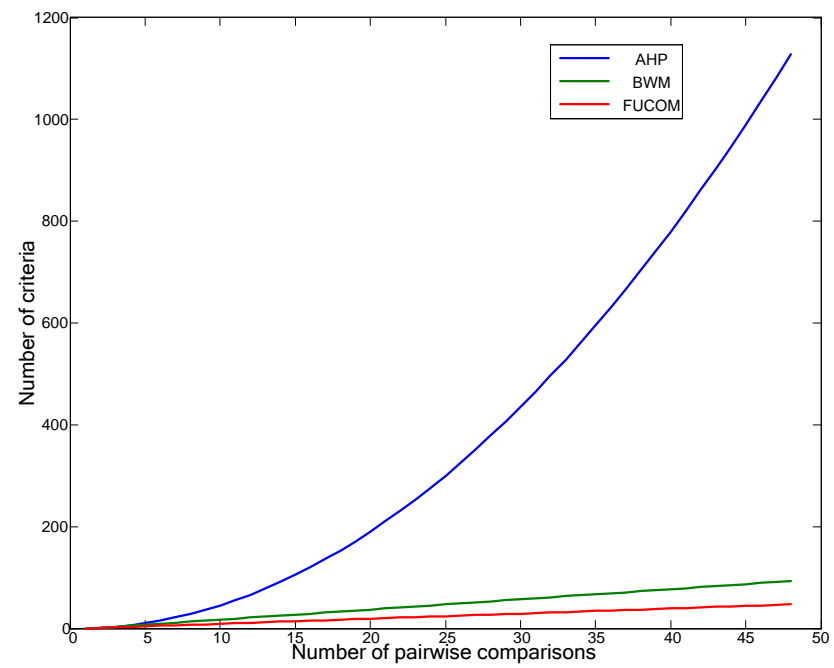

Fig. 1 Number of pairwise comparison of different weighting methods 
Badi and Abdulshahed [13] showed the application of the FUCOM to evaluation of the line in air traffic. Noureddine and Ristic [14] used a hybrid FUCOM-MABAC model for evaluating transport routes for dangerous goods in road traffic. In addition to these studies, the FUCOM was applied to supply chain management $[15,16]$.

In the real world, it often happens that, due to partial knowledge of attributes or the lack of information regarding the problem, decision-makers prefer to evaluate attributes using linguistic variables instead of crisp values. In such situations, information on the attributes obtained from decision-makers may be unclear, imprecise or incomplete. The fuzzy set theory introduced by [17] is one of the tools successfully used to present such inaccuracies in a mathematical form. Since the creation of fuzzy sets, MCDM problems with imprecise information have been successfully modeled using the theory of fuzzy sets. According to the best knowledge of the authors, the application of the FUCOM in the fuzzy environment has not yet been shown and this paper is targeted at filling this gap in the literature. Thus, it is one of the motives for creating this extension of the FUCOM's work in the fuzzy environment. Therefore, the aims of this paper are as follows.

- To improve the methodology for defining the weight coefficients of criteria by developing a FUCOM-F.

- To determine the weights of criteria using the FUCOM throughout a detailed algorithm in the fuzzy environment.

- To bridge the gap that exists in the methodology for determining the weight coefficients of criteria throughout a new model in treating uncertainty, which is based on fuzzy numbers.

To achieve this, the rest of the paper was organized as follows. The proposed FUCOM-F model was introduced in detail in the next section. In the third section of the paper, an illustrative example was conducted to reveal the steps of the proposed method. At the same time, the results obtained from comparisons of three models i.e. FUCOM-F, FBWM, and FAHP were discussed. Finally, conclusions are presented in Section 4.

\section{FUZZY FULL CONSISTENCY MCDM METHOD}

In this section, the FUCOM-F has been discussed in detail after giving information on triangular fuzzy numbers (TFNs) which are the expressions of linguistic variables as fuzzy numbers.

\subsection{Triangular fuzzy numbers}

The Fuzzy set theory assigns membership degrees to linguistic variables and considers them as probability distribution. To achieve this, it utilizes fuzzy numbers. Although there are various shapes of fuzzy numbers like trapezoidal, triangular or Gaussian, the TFN is the most preferred by researchers in literature [18]. The outlines of fuzzy sets and TFNs have been given below briefly $[18,19,20]$.

Definition 1: A fuzzy number is a special fuzzy set $F=\left\{\left(x, \mu_{F}(x)\right), x \in \mathfrak{R}\right\}$, where $x$ takes its values on the real line, $\mathfrak{R}:-\infty \leq x \leq \infty$ and $\mu_{F}(x)$ is a membership function in the closed interval $[0,1]$. 
Definition 2: A TFN expresses the relative strength of each pair of elements in the same hierarchy, and can be denoted as $T=(l, m, u)$, where $l \leq m \leq u$. Parameters $l, m, u$ indicate the lower bound value, the center, and the upper bound value in a fuzzy event, respectively. Triangular type membership function of $T$ fuzzy number can be described as in Eq. (1)

$$
\mu_{T}(x)= \begin{cases}0 & , x<l \\ (x-l) /(m-l) & , l \leq x \leq m \\ (u-x) /(u-m) & , m \leq x \leq u \\ 0 & , x>u\end{cases}
$$

Consider two TFNs $T_{1}=\left(l_{1}, m_{1}, u_{1}\right)$ and $T_{2}=\left(l_{2}, m_{2}, u_{2}\right)$. The following describes the basic operations of two fuzzy numbers, $T_{1}$ and $T_{2}$, respectively:

$$
\begin{gathered}
\left(l_{1}, m_{1}, u_{1}\right) \oplus\left(l_{2}, m_{2}, u_{2}\right)=\left(l_{1}+l_{2}, m_{1}+m_{2}, u_{1}+u_{2}\right) \\
\left(l_{1}, m_{1}, u_{1}\right) \otimes\left(l_{2}, m_{2}, u_{2}\right)=\left(l_{1} l_{2}, m_{1} m_{2}, u_{1} u_{2}\right) \\
\left(l_{1}, m_{1}, u_{1}\right) /\left(l_{2}, m_{2}, u_{2}\right) \cong\left(l_{1} / u_{2}, m_{1} / m_{2}, u_{1} / l_{2}\right) \text { for } l_{i}>0, m_{i}>0, u_{i}>0 \\
\left(l_{i}, m_{i}, u_{i}\right)^{-1} \approx\left(\frac{1}{u_{i}}, \frac{1}{m_{i}}, \frac{1}{l_{i}}\right) \text { for } l_{i}>0, m_{i}>0, u_{i}>0
\end{gathered}
$$

Definition 3: The graded mean integration representation (GMIR)

Let $a_{j}=\left(l_{j}, m_{j}, u_{j}\right)$ be a TFN and GMIR $R\left(a_{j}\right)$ of $a_{i}$ can be calculated as:

$$
R\left(a_{j}\right)=\frac{l_{j}+4 m_{j}+u_{j}}{6}
$$

\subsection{Fuzzy FUCOM (FUCOM-F)}

Assume that in a MCDM problem, there are $n$ evaluation criteria that are denoted as $w_{j}, j=1,2, \ldots, n$, and their weight coefficients need to be determined. Subjective models for determining weights based on pairwise comparison of criteria require decision-makers to determine the degree of impact of criterion $i$ on criterion $j$. The degree of influence criterion $i$ has on criterion $j$ is presented as the value of comparison $\left(a_{i j}\right)$. Since the obtained values of comparison $a_{i j}$ are not based on accurate measurements, but on subjective estimates, it is expected that existing uncertainties will be presented with fuzzy numbers. In the application of fuzzy numbers in the MCDM models, linguistic scales are most frequently used. Thus, throughout this paper, a fuzzy linguistic scale [20], described by triangular fuzzy numbers, is used to present expert preferences in the FUCOM-F (Table 1). Because it is a multi-criteria model, it should be emphasized that the model 
presented can be also used to determine the weight coefficients of alternatives, and, therefore, the final rank and the selection of the optimum one from the set of alternatives observed.

Table 1 Fuzzy linguistic scale [20]

\begin{tabular}{lc}
\hline Linguistic terms & Membership function \\
\hline Equally important (EI) & $(1,1,1)$ \\
Weakly important (WI) & $(2 / 3,1,3 / 2)$ \\
Fairly Important (FI) & $(3 / 2,2,5 / 2)$ \\
Very important (VI) & $(5 / 2,3,7 / 2)$ \\
Absolutely important (AI) & $(7 / 2,4,9 / 2)$ \\
\hline
\end{tabular}

Based on the main settings of the FUCOM [9], the extension of the traditional model in a fuzzy environment has been carried out. Accordingly, the FUCOM-F algorithm has been presented in detail in four steps.

Step 1 Determine the decision criteria. An initial step in multi-criteria models for evaluating alternatives is defining a set of evaluation criteria. As defined in the beginning of this chapter, supposing that there are $n(j=1,2, \ldots, n)$ evaluation criteria that are represented by a set $C=\left\{C_{1}, C_{2}, \ldots, C_{n}\right\}$.

Step 2 Rank the decision criteria. Experts determine the rank of criteria in accordance with their preferences regarding the significance of the criteria. The first rank is assigned to a criterion that is expected to have the highest weight coefficient and so on, towards the criterion of the least significance. The last place is held by the criterion for which we expect to have the lowest value of the weight coefficient. Thus, the criteria ranked according to the expected impact on decision-making in a MCDM model is obtained.

$$
C_{j(1)}>C_{j(2)}>\ldots>C_{j(k)}
$$

where $k$ represents the rank of the criterion observed. If two or more criteria have the same ranking, the equality sign is placed between the criteria instead of ">".

Step 3 Comparisons of the criteria using TFNs. The criteria are compared to each other using fuzzy linguistic expressions from a defined scale (Table 1). The comparison is made with respect to the first-ranked (most significant) criterion. Thus, we obtain the fuzzy criterion significance $\left(\varpi_{C_{j(k)}}\right.$ ) for all the criteria that are ranked in Step 2. Since the first-ranked criterion is compared with itself (its significance is $\varpi_{C_{j(1)}}=E I$ ), $n-1$ comparison of the remaining criteria must be performed. Based on the defined significance of criteria, fuzzy comparative significance $\varphi_{k /(k+1)}$ is determined by applying Eq. (8).

$$
\varphi_{k /(k+1)}=\frac{\varpi_{C_{j(k+1)}}}{\varpi_{C_{j(k)}}}=\frac{\left(\varpi_{C_{j(k+1)}}^{l}, \varpi_{C_{j(k+1)}^{m}}^{m}, \varpi_{C_{j(k+1)}}^{u}\right)}{\left(\varpi_{C_{j(k)}}^{l}, \varpi_{C_{j(k)}}^{m}, \varpi_{C_{j(k)}}^{u}\right)}
$$


Thus, a fuzzy vector of comparative significance of the evaluation criteria is obtained using Eq. (9).

$$
\Phi=\left(\varphi_{1 / 2}, \varphi_{2 / 3}, \ldots, \varphi_{k /(k+1)}\right)
$$

where $\varphi_{k /(k+1)}$ represents the significance that the criterion of $C_{j(k)}$ rank has in relation to the criterion of $C_{j(k+1)}$ rank.

Step 4 Calculate the optimal fuzzy weights. In the fourth step, the final values of the fuzzy weight coefficients of criteria $\left(w_{1}, w_{2}, \ldots, w_{n}\right)^{T}$ are calculated. The final values of weight coefficients should satisfy two conditions:

Condition 1 The ratio of weight coefficients of the observed criteria $\left(C_{j(k)}\right.$ and $\left.C_{j(k+1)}\right)$ should be equal to their comparative significance $\left(\varphi_{k /(k+1)}\right)$ defined in Step 2, i.e. that it fulfills the condition:

$$
\frac{w_{k}}{w_{k+1}}=\varphi_{k /(k+1)}
$$

Condition 2 In addition to the condition defined by expression (9), the final values of weight coefficients should satisfy transitivity, i.e. that $\varphi_{k /(k+1)} \otimes \varphi_{(k+1) /(k+2)}=\varphi_{k /(k+2)}$, i.e. that $\frac{w_{k}}{w_{k+1}} \otimes \frac{w_{k+1}}{w_{k+2}}=\frac{w_{k}}{w_{k+2}}$. Thus, another condition that needs to be satisfied by the final values of weight coefficients is obtained:

$$
\frac{w_{k}}{w_{k+2}}=\varphi_{k /(k+1)} \otimes \varphi_{(k+1) /(k+2)}
$$

Minimum DMC, i.e. $\chi=0$, is satisfied only if the transitivity among weight coefficients is completely satisfied. Then, it can be said that $\frac{w_{k}}{w_{k+1}}-\varphi_{k /(k+1)}=0$ and $\frac{w_{k}}{w_{k+2}}-\varphi_{k /(k+1)} \otimes \varphi_{(k+1) /(k+2)}=0$. For such obtained values of weight coefficients, DMC is $\chi=0$. In order to satisfy these conditions, it is necessary to determine the values of the weight coefficients of criteria $\left(w_{1}, w_{2}, \ldots, w_{n}\right)^{T}$ that satisfy the condition that $\left|\frac{w_{k}}{w_{k+1}}-\varphi_{k /(k+1)}\right| \leq \chi$ and $\left|\frac{w_{k}}{w_{k+2}}-\varphi_{k /(k+1)} \otimes \varphi_{(k+1) /(k+2)}\right| \leq \chi$ with the minimization of value $\chi$.

Based on the settings defined, the final nonlinear model for determining the optimal fuzzy values of the weight coefficients of the evaluation criteria can be $\operatorname{set}\left(w_{1}, w_{2}, \ldots, w_{n}\right)^{T}$. 


$$
\begin{aligned}
& \min \chi \\
& \text { s.t. } \\
& \mid \begin{array}{l}
\left|\frac{w_{k}}{w_{k+1}}-\varphi_{k /(k+1)}\right| \leq \chi, \forall j \\
\left|\frac{w_{k}}{w_{k+2}}-\varphi_{k /(k+1)} \otimes \varphi_{(k+1) /(k+2)}\right| \leq \chi, \forall j \\
\sum_{j=1}^{n} w_{j}=1, \forall j, \\
w_{j}^{l} \leq w_{j}^{m} \leq w_{j}^{u}, \\
w_{j}^{l} \geq 0, \forall j \\
j=1,2, \ldots, n
\end{array}
\end{aligned}
$$

where $w_{j}=\left(w_{j}^{l}, w_{j}^{m}, w_{j}^{u}\right)$ and $\varphi_{k /(k+1)}=\left(\varphi_{k /(k+1)}^{l}, \varphi_{k /(k+1)}^{m}, \varphi_{k /(k+1)}^{u}\right)$.

In order to achieve the highest consistency, it is necessary to satisfy the condition that $\frac{w_{k}}{w_{k+1}}-\varphi_{k /(k+1)}=0$ and $\frac{w_{k}}{w_{k+2}}-\varphi_{k /(k+1)} \otimes \varphi_{(k+1) /(k+2)}=0$. Thereby, the model given by Eq. (12) can be transformed into a fuzzy linear model, Eq. (13). The optimal fuzzy values of weight coefficients are obtained $\left(w_{1}, w_{2}, \ldots, w_{n}\right)^{T}$, if it is solved.

$$
\begin{aligned}
& \min \chi \\
& \text { s.t. } \\
& \left\{\begin{array}{l}
\left|w_{k}-w_{k+1} \otimes \varphi_{k /(k+1)}\right| \leq \chi, \forall j \\
\left|w_{k}-w_{k+2} \otimes \varphi_{k /(k+1)} \otimes \varphi_{(k+1) /(k+2)}\right| \leq \chi, \forall j \\
\sum_{j=1}^{n} w_{j}=1, \forall j, \\
w_{j}^{l} \leq w_{j}^{m} \leq w_{j}^{u}, \\
w_{j}^{l} \geq 0, \forall j \\
j=1,2, \ldots, n
\end{array}\right.
\end{aligned}
$$

where $w_{j}=\left(w_{j}^{l}, w_{j}^{m}, w_{j}^{u}\right)$ and $\varphi_{k /(k+1)}=\left(\varphi_{k /(k+1)}^{l}, \varphi_{k /(k+1)}^{m}, \varphi_{k /(k+1)}^{u}\right)$.

\section{ILLUSTRATIVE EXAMPLE}

This section of the paper presents the application of the FUCOM-F using an example of determining the weight coefficients of the criteria for evaluating green suppliers. With its application in the example shown, the model verification and comparison of results with other models from the literature, i.e. fuzzy BWM (FBWM) [20] and fuzzy AHP (FAHP) [21] models, have been performed. 
Based on a literature analysis, 15 representative criteria have been identified for the evaluation of green suppliers, Table 2 . The criteria are grouped within three dimensions: economic $\left(C_{1}\right)$, environmental $\left(C_{2}\right)$, and social $\left(C_{3}\right)$. As can be seen in Table 2 , the criteria are arranged at two hierarchical levels.

Table 2 Dimensions and their factors for evaluating green suppliers

\begin{tabular}{lll}
\hline Dimension & Criteria & Code \\
\hline & Cost/price & C11 \\
& Quality & $\mathrm{C} 12$ \\
Economic (C1) & Delivery & $\mathrm{C} 13$ \\
& Technology & $\mathrm{C} 14$ \\
& Flexibility & $\mathrm{C} 15$ \\
& Financial capability & $\mathrm{C} 16$ \\
\hline & Pollution production & $\mathrm{C} 21$ \\
Environmental (C2) & Eco-design & $\mathrm{C} 22$ \\
& Environmental management system & $\mathrm{C} 23$ \\
& Green image & $\mathrm{C} 24$ \\
& Environmental training & $\mathrm{C} 25$ \\
\hline & Social responsibility & $\mathrm{C} 31$ \\
Social (C3) & Commitment to health and safety of employees & $\mathrm{C} 32$ \\
& Ethical issues & $\mathrm{C} 33$ \\
& The interests and rights of employee & $\mathrm{C} 34$ \\
\hline
\end{tabular}

The first level includes economic, environmental, and social dimensions. The second level is presented by the groups of criteria within $C_{1}, C_{2}$, and $C_{3}$. The aim of the FUCOM-F application is to determine the global values of weight coefficients of the second-level criteria. The solution of this problem using the FUCOM-F is performed by defining four models:

Model 1 - Determining the local values of weight coefficients of $C_{1}, C_{2}$ and $C_{3}$,

Model 2 - Determining the local values of weight coefficients within the $C_{1}$,

Model 3 - Determining the local values of weight coefficients within the $C_{2}$ and,

Model 4 - Determining the local values of weight coefficients within the $C_{3}$.

By multiplying the local values of the weight coefficients of dimensions with corresponding local values of the criteria (within the observed dimension), the global optimal values of the weights of the criteria are obtained. A detailed overview of Models 1-4 is presented in the next sub-section.

\subsection{Determining the fuzzy weights}

\subsubsection{Model 1 - Weight coefficients of $C_{1}, C_{2}$, and $C_{3}$ dimensions}

After defining the first-level criteria, in the second step their ranking was performed. Dimensions were ranked as follows: Environmental $\left(C_{1}\right)>\operatorname{Economic}\left(C_{2}\right)>\operatorname{Social}\left(C_{3}\right)$. In the next step (Step 3), based on the preferences of decision-makers, the linguistic variables of the comparative significance of the criteria ranked were determined (Table 3).

Table 3 Linguistic evaluations of main dimensions

\begin{tabular}{llll}
\hline Dimensions & $C_{1}$ & $C_{2}$ & $C_{3}$ \\
\hline Linguistic variables & EI & WI & FI \\
\hline
\end{tabular}


By applying the fuzzy linguistic scale, linguistic variables were transformed into TFNs, shown in Table 4.

Table 4 TFN transformations of evaluations

\begin{tabular}{lccc}
\hline Dimensions & $C_{1}$ & $C_{2}$ & $C_{3}$ \\
\hline TFN & $(1,1,1)$ & $(2 / 3,1,3 / 2)$ & $(3 / 2,2,5 / 2)$ \\
\hline
\end{tabular}

Applying expression (8), the comparative significance of the criteria has been defined as follows.

$$
\begin{gathered}
\varphi_{C 1 / C 2}=\varpi_{C 1} / \varpi_{C 2}=(2 / 3,1,3 / 2) /(1,1,1)=(2 / 3,1,3 / 2) \\
\varphi_{C 2 / C 3}=\varpi_{C 2} / \varpi_{C 3}=(3 / 2,2,5 / 2) /(2 / 3,1,3 / 2)=(1.00,2.00,3.73)
\end{gathered}
$$

By calculating the comparative significance of the criteria, the vector of comparative significance $\Phi=((0.67,1.00,1.50),(1.00,2.00,3.73))$ was defined. In the following section (Step 4), the constraints of the Model (12) were defined based on the vector of comparative significance. By applying expression (10), we have defined the first group of constraints: $w_{C 1} / w_{C 2}=(2 / 3,1,3 / 2)$ and $w_{C 2} / w_{C 3}=(1.00,2.00,3.73)$. Based on expression (11), a constraint that arises from the conditions of relation transitivity $w_{C 1} / w_{C 3}=$ $(0.67,1.00,1.50) \cdot(1.00,2.00,3.73)=(0.67,2.00,5.60)$ was defined. Based on the constraints defined, a model (13) for determining the optimal values of the weight coefficients of dimensions was formed.

By solving the model, the optimum local values of the weight coefficients:

$$
w_{j}=((0.261,0.3891,0.5831),(0.3881,0.3881,0.3881),(0.1038,0.1945,0.3891))^{T}
$$

and $\chi=0.001$ were obtained (Fig. 2). The Lingo 17.0 software has been used to solve the model presented.

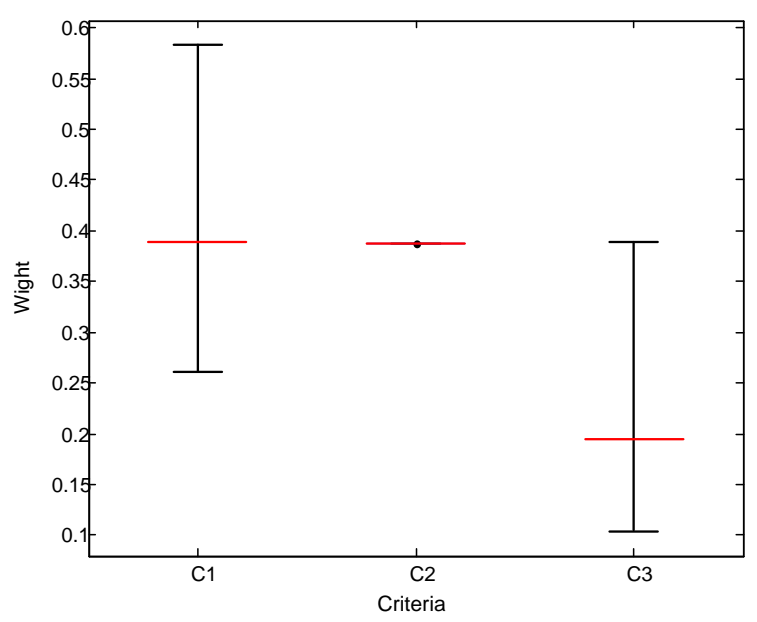

Fig. 2 Fuzzy criteria weights for Model 1 


\subsubsection{Model 2 - Weight coefficients within the $C_{1}$ dimension}

A similar methodology was also applied to solving Model 2. After defining the criteria within the $C_{1}$ dimension, the ranking of criteria was performed: $C_{11}>C_{13}>C_{15}>C_{16}>$ $C_{12}>C_{14}$. In the next step, the comparison of the criteria was performed (Table 5).

Table 5 Linguistic evaluations of economic factors

\begin{tabular}{lcccccc}
\hline Factors & $C 11$ & $C 13$ & $C 15$ & $C 16$ & $C 12$ & $C 14$ \\
\hline Linguistic variables & EI & WI & FI & VI & AI & AI \\
\hline
\end{tabular}

Then, the comparative significance of the criteria was defined as follows.

$$
\begin{gathered}
\varphi_{C 11 / C 13}=(2 / 3,1,3 / 2) /(1,1,1)=(2 / 3,1,3 / 2), \\
\varphi_{C 13 / C 15}=(3 / 2,2,5 / 2) /(2 / 3,1,3 / 2)=(1.0,2.0,3.73), \\
\varphi_{C 15 / C 16}=(5 / 2,3,7 / 2) /(3 / 2,2,5 / 2)=(1.0,1.5,2.33), \\
\varphi_{C 16 / C 12}=(7 / 2,4,9 / 2) /(5 / 2,3,7 / 2)=(1.0,1.33,1.8), \\
\varphi_{C 12 / C 14}=(7 / 2,4,9 / 2) /(7 / 2,4,9 / 2)=(0.78,1.0,1.29)
\end{gathered}
$$

Based on the comparative significance of the criteria, a vector of comparative significance was defined as

$$
\Phi=((0.67,1,1.5),(1,2,3.73),(1,1.5,2.33),(1,1.33,1.8),(0.78,1,1.29))
$$

and by applying expression (10), the first group of constraints of the fuzzy linear model was defined as

$$
\begin{gathered}
w_{C 1} / w_{C 2}=(0.67,1,1.5), w_{C 3} / w_{C 5}=(1.0,2.0,3.73), w_{C 5} / w_{C 6}=(1.0,1.5,2.33), \\
w_{C 6} / w_{C 2}=(1.0,1.33,1.8) \text { and } w_{C 2} / w_{C 4}=(0.78,1.0,1.29)
\end{gathered}
$$

By applying Eq. (11), the second group of constraints was defined as

$$
\begin{gathered}
w_{C 1} / w_{C 5}=(0.67,2.0,5.6), w_{C 3} / w_{C 6}=(1.0,3.0,8.71), w_{C 5} / w_{C 2}=(1.0,2.0,4.2), \\
\text { and } w_{C 6} / w_{C 4}=(0.78,1.33,2.31) .
\end{gathered}
$$

The optimum values of the criteria were obtained by solving the fuzzy linear model presented below.

By solving the above model with Lingo 17.0, the weight coefficients of the criteria within the $C_{1}$ dimension with a deviation from the maximum consistency $\chi=0.05$ were obtained. 


$$
w_{j}(C 1)=\left(\begin{array}{c}
(0.1525,0.3100,0.3125), \\
(0.0479,0.0939,0.0992), \\
(0.1726,0.2970,0.2975), \\
(0.0747,0.1200,0.1204), \\
(0.0654,0.1475,0.1475), \\
(0.0403,0.1170,0.1190)
\end{array}\right) .
$$

\subsubsection{Model 3 - Weight coefficients within the $C_{2}$ dimension}

Within the second dimension $\left(C_{2}\right)$, five criteria have been identified which, based on expert preferences, were ranked as follows: $C_{22}>C_{21}>C_{24}>C_{25}>C_{23}$. Based on the preferences of decision-makers, the linguistic values of the comparative significance of the criteria ranked have been determined (Table 6).

Table 6 Linguistic evaluations of environmental factors

\begin{tabular}{lccccc}
\hline Factors & C22 & C21 & C24 & C25 & C23 \\
\hline Linguistic variables & EI & WI & WI & FI & AI \\
\hline
\end{tabular}

In the next step, based on expert comparisons of the criteria (Table 6), using Eq. (8), the comparative significance of the criteria $\varphi_{C 22 / C 21}=(0.67,1.0,1.5), \varphi_{C 21 / C 24}=(0.45,1.0,224)$, $\varphi_{C 24 / C 25}=(1.67,3.0,5.2), \varphi_{C 25 / C 23}=(1.0,1.3,1.8)$, and a vector of comparative significance

$$
\Phi=((0.67,1.0,1.5),(0.45,1.0,2.24),(1.67,3.0,5.2),(1.0,1.3,1.8))
$$

were defined. From vector $\Phi$, applying Eq. (10), the first group of the constraints of the fuzzy linear model was defined as

$$
\begin{gathered}
w_{C 22} / w_{C 21}=(0.67,1.0,1.5), w_{C 21} / w_{C 24}=(0.45,1.0,2.24), \\
w_{C 24} / w_{C 25}=(1.67,3.0,5.2), w_{C 25} / w_{C 23}=(1.0,1.3,1.8),
\end{gathered}
$$

while by applying Eq. (11), the second group of constraints was defined as

$$
w_{C 22} / w_{C 24}=(0.3,1.0,3.36), w_{C 21} / w_{C 25}=(0.74,3.0,11.7), w_{C 24} / w_{C 23}=(1.67,4.0,9.4) \text {. }
$$

The optimum values of the criteria within the $C_{2}$ dimension were obtained by solving the following fuzzy linear model. 
Prioritizing the Weights of the Evaluation Criteria Under Fuzziness: the Fuzzy Full Consistency Method... 431

$$
\begin{aligned}
& \min \chi \\
& \text { s.t. } \\
& \left\{\begin{array}{lll}
\left(w_{2}^{l}-w_{1}^{u} \cdot 0.67\right) \leq \chi ; & \left(w_{4}^{m}-w_{5}^{m} \cdot 3\right) \leq \chi ; & \left(w_{2}^{u}-w_{4}^{l} \cdot 3.36\right) \geq-\chi ; \\
\left(w_{2}^{l}-w_{1}^{u} \cdot 0.67\right) \geq-\chi ; & \left(w_{4}^{m}-w_{5}^{m} \cdot 3\right) \geq-\chi ; & \left(w_{1}^{l}-w_{5}^{u} \cdot 0.74\right) \leq \chi ; \\
\left(w_{2}^{m}-w_{1}^{m}\right) \leq \chi ; & \left(w_{4}^{u}-w_{5}^{l} \cdot 5.2\right) \leq \chi ; & \left(w_{1}^{l}-w_{5}^{u} \cdot 0.74\right) \geq-\chi ; \\
\left(w_{2}^{m}-w_{1}^{m}\right) \geq-\chi ; & \left(w_{4}^{u}-w_{5}^{l} \cdot 5.2\right) \geq-\chi ; & \left(w_{1}^{m}-w_{5}^{m} \cdot 3\right) \leq \chi ; \\
\left(w_{2}^{u}-w_{1}^{l} \cdot 1.5\right) \leq \chi ; & \left(w_{5}^{l}-w_{3}^{u}\right) \leq \chi ; & \left(w_{1}^{m}-w_{5}^{m} \cdot 3\right) \geq-\chi ; \\
\left(w_{2}^{u}-w_{1}^{l} \cdot 1.5\right) \geq-\chi ; & \left(w_{5}^{l}-w_{3}^{u}\right) \geq-\chi ; & \left(w_{1}^{u}-w_{5}^{l} \cdot 11.7\right) \leq \chi ; \\
\left(w_{1}^{l}-w_{4}^{u} \cdot 0.45\right) \leq \chi ; & \left(w_{5}^{m}-w_{3}^{m} \cdot 1.33\right) \leq \chi ; & \left(w_{1}^{u}-w_{5}^{l} \cdot 11.7\right) \geq-\chi ; \\
\left(w_{1}^{l}-w_{4}^{u} \cdot 0.45\right) \geq-\chi ; & \left(w_{5}^{m}-w_{3}^{m} \cdot 1.33\right) \geq-\chi ; & \left(w_{4}^{l}-w_{3}^{u} \cdot 1.67\right) \leq \chi ; \\
\left(w_{1}^{m}-w_{4}^{m}\right) \leq \chi ; & \left(w_{5}^{u}-w_{3}^{l} \cdot 1.8\right) \leq \chi ; & \left(w_{4}^{l}-w_{3}^{u} \cdot 1.67\right) \geq-\chi ; \\
\left(w_{1}^{m}-w_{4}^{m}\right) \geq-\chi ; & \left(w_{5}^{u}-w_{3}^{l} \cdot 1.8\right) \geq-\chi ; & \left(w_{4}^{m}-w_{3}^{m} \cdot 4\right) \leq \chi ; \\
\left(w_{1}^{u}-w_{4}^{l} \cdot 2.24\right) \leq \chi ; & \left(w_{2}^{l}-w_{4}^{u} \cdot 0.3\right) \leq \chi ; & \left(w_{4}^{m}-w_{3}^{m} \cdot 4\right) \geq-\chi ; \\
\left(w_{1}^{u}-w_{4}^{l} \cdot 2.24\right) \geq-\chi ; & \left(w_{2}^{l}-w_{4}^{u} \cdot 0.3\right) \geq-\chi ; & \left(w_{4}^{u}-w_{3}^{l} \cdot 9.4\right) \leq \chi ; \\
\left(w_{4}^{l}-w_{5}^{u} \cdot 1.67\right) \leq \chi ; & \left(w_{2}^{m}-w_{4}^{m}\right) \leq \chi ; & \left(w_{4}^{u}-w_{3}^{l} \cdot 9.4\right) \geq-\chi ; \\
\left(w_{4}^{l}-w_{5}^{u} \cdot 1.67\right) \geq-\chi ; & \left(w_{2}^{u}-w_{4}^{l} \cdot 3.36\right) \leq \chi ; & \\
\left(w_{1}^{l}+4 \cdot w_{1}^{m}+w_{1}^{u}\right) / 6+\left(w_{2}^{l}+4 \cdot w_{2}^{m}+w_{2}^{u}\right) / 6+\left(w_{3}^{l}+4 \cdot w_{3}^{m}+w_{3}^{u}\right) / 6 \\
+\left(w_{4}^{l}+4 \cdot w_{4}^{m}+w_{4}^{u}\right) / 6+\left(w_{5}^{l}+4 \cdot w_{5}^{m}+w_{5}^{u}\right) / 6=1 ; \\
w_{1}^{l} \leq w_{1}^{m} \leq w_{1}^{u} ; w_{2}^{l} \leq w_{2}^{m} \leq w_{2}^{u} ; w_{3}^{l} \leq w_{3}^{m} \leq w_{3}^{u} ; & \\
w_{4}^{l} \leq w_{4}^{m} \leq w_{4}^{u} ; w_{5}^{l} \leq w_{5}^{m} \leq w_{5}^{u} ; & \\
w_{1}^{l}, w_{2}^{l}, w_{3}^{l}, w_{4}^{l}, w_{5}^{l} \geq 0 . &
\end{array}\right.
\end{aligned}
$$

If the above model is solved, the weight coefficients of the criteria within the $C_{2}$ dimension with a deviation from the maximum consistency $\chi=0.07$ are obtained.

$$
w_{j}(C 2)=\left(\begin{array}{c}
(0.1589,0.3314,0.3332), \\
(0.1517,0.3100,0.3131), \\
(0.0353,0.0828,0.1096), \\
(0.1154,0.2567,0.2567), \\
(0.0349,0.1105,0.1138)
\end{array}\right)
$$

\subsubsection{Model 4 - Weight coefficient within the $C_{3}$ dimension}

In Model 4 within the third dimension $\left(C_{3}\right)$, four criteria have been identified. The criteria were ranked on the basis of expert preferences $C_{34}>C_{31}>C_{32}>C_{33}$ and the comparison of the criteria was made using TFNs. The linguistic variables of the comparative significance of the criteria ranked are shown in Table 7. 
Table 7 Linguistic evaluations of social factors

\begin{tabular}{lcccc}
\hline Factors & C34 & C31 & C32 & C33 \\
\hline Linguistic variables & EI & WI & FI & VI \\
\hline
\end{tabular}

Therefore, based on expert comparisons of the criteria, the comparative significance of the criteria

$$
\varphi_{C 34 / C 31}=(0.67,1.0,1.5), \varphi_{C 31 / C 32}=(1.0,2.0,3.73), \varphi_{C 32 / C 33}=(1.0,1.5,2.3),
$$

and a vector of comparative significance

$$
\Phi=((0.67,1.0,1.5),(1.0,2.0,3.7),(1.0,1.5,2.3))
$$

were defined. From vector $\Phi$, by applying Eqs. (10) and (11), two groups of constraints were defined: first group:

$$
w_{C 34} / w_{C 31}=(0.67,1.0,1.5), w_{C 31} / w_{C 32}=(1.0,2.0,3.73), w_{C 32} / w_{C 33}=(1.0,1.5,2.3)
$$

and second group:

$$
w_{C 34} / w_{C 32}=(0.67,2.0,5.6) \text { and } w_{C 31} / w_{C 33}=(1.0,3.0,8.7) \text {. }
$$

The optimum values of the criteria within the $C_{3}$ dimension were obtained by solving the following fuzzy linear model.

$$
\begin{aligned}
& \min \chi \\
& \text { s.t. } \\
& \left\{\begin{array}{lll}
\left(w_{4}^{l}-w_{1}^{u} \cdot 0.67\right) \leq \chi ; & \left(w_{1}^{u}-w_{2}^{l} \cdot 3.7\right) \leq \chi ; & \left(w_{4}^{m}-w_{2}^{m} \cdot 2\right) \leq \chi ; \\
\left(w_{4}^{l}-w_{1}^{u} \cdot 0.67\right) \geq-\chi ; & \left(w_{1}^{u}-w_{2}^{l} \cdot 3.7\right) \geq-\chi ; & \left(w_{4}^{m}-w_{2}^{m} \cdot 2\right) \geq-\chi ; \\
\left(w_{4}^{m}-w_{1}^{m}\right) \leq \chi ; & \left(w_{2}^{l}-w_{3}^{u}\right) \leq \chi ; & \left(w_{4}^{u}-w_{2}^{l} \cdot 5.6\right) \leq \chi ; \\
\left(w_{4}^{m}-w_{1}^{m}\right) \geq-\chi ; & \left(w_{2}^{l}-w_{3}^{u}\right) \geq-\chi ; & \left(w_{4}^{u}-w_{2}^{l} \cdot 5.6\right) \geq-\chi ; \\
\left(w_{4}^{u}-w_{1}^{l} \cdot 1.5\right) \leq \chi ; & \left(w_{2}^{m}-w_{3}^{m} \cdot 1.5\right) \leq \chi ; & \left(w_{1}^{l}-w_{3}^{u}\right) \leq \chi ; \\
\left(w_{4}^{u}-w_{1}^{l} \cdot 1.5\right) \geq-\chi ; & \left(w_{2}^{m}-w_{3}^{m} \cdot 1.5\right) \geq-\chi ; & \left(w_{1}^{l}-w_{3}^{u}\right) \geq-\chi ; \\
\left(w_{1}^{l}-w_{2}^{u}\right) \leq \chi ; & \left(w_{2}^{u}-w_{3}^{l} \cdot 2.3\right) \leq \chi ; & \left(w_{1}^{m}-w_{3}^{m} \cdot 3\right) \leq \chi ; \\
\left(w_{1}^{l}-w_{2}^{u}\right) \geq-\chi ; & \left(w_{2}^{u}-w_{3}^{l} \cdot 2.3\right) \geq-\chi ; & \left(w_{1}^{m}-w_{3}^{m} \cdot 3\right) \geq-\chi ; \\
\left(w_{1}^{m}-w_{2}^{m} \cdot 2\right) \leq \chi ; & \left(w_{4}^{l}-w_{2}^{u} \cdot 0.67\right) \leq \chi ; & \left(w_{1}^{u}-w_{3}^{l} \cdot 8.7\right) \leq \chi ; \\
\left(w_{1}^{m}-w_{2}^{m} \cdot 2\right) \geq-\chi ; & \left(w_{4}^{l}-w_{2}^{u} \cdot 0.67\right) \geq-\chi ; & \left(w_{1}^{u}-w_{3}^{l} \cdot 8.7\right) \geq-\chi ; \\
\left(w_{1}^{l}+4 \cdot w_{1}^{m}+w_{1}^{u}\right) / 6+\left(w_{2}^{l}+4 \cdot w_{2}^{m}+w_{2}^{u}\right) / 6+\left(w_{3}^{l}+4 \cdot w_{3}^{m}+w_{3}^{u}\right) / 6+ \\
+\left(w_{4}^{l}+4 \cdot w_{4}^{m}+w_{4}^{u}\right) / 6=1 ;
\end{array}\right. \\
& w_{1}^{l} \leq w_{1}^{m} \leq w_{1}^{u} ; w_{2}^{l} \leq w_{2}^{m} \leq w_{2}^{u} ; \\
& w_{3}^{l} \leq w_{3}^{m} \leq w_{3}^{u} ; w_{4}^{l} \leq w_{4}^{m} \leq w_{4}^{u} ; \\
& w_{1}^{l}, w_{2}^{l}, w_{3}^{l}, w_{4}^{l} \geq 0 .
\end{aligned}
$$


If the above model is solved, the weight coefficients of the criteria within the $C_{3}$ dimension with a deviation from the maximum consistency $\chi=0.06$ are obtained.

$$
w_{j}(C 3)=\left(\begin{array}{c}
(0.2155,0.3698,0.3714), \\
(0.0816,0.1842,0.1842), \\
(0.0503,0.1461,0.1486), \\
(0.1904,0.3900,0.3902)
\end{array}\right)
$$

As previously mentioned, for the evaluation of green suppliers in a multi-criteria model, the weight coefficients of the second-level criteria were used. Since the criteria were divided into two hierarchical levels, the values of the criteria by the hierarchical levels represent local fuzzy values. The global values of the weights of the criteria were defined by multiplying the weight coefficients of the first hierarchical level with the groups of criteria of the second hierarchical level. The final global fuzzy values of the weights of the criteria are presented in Fig. 3.

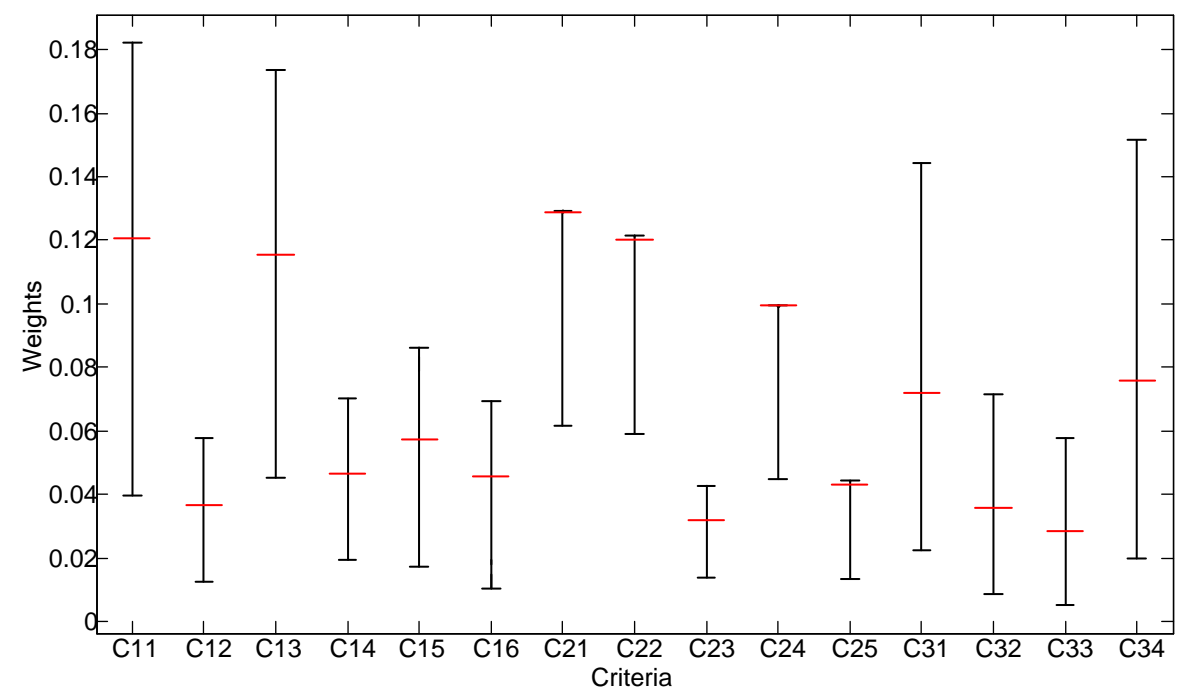

Fig. 3 Final fuzzy values of the criteria

The validation of the results was performed by comparing the results obtained with the application of the FBWM and FAHP models. For the research shown in this paper, all data were collected in the form of fuzzy matrices of pairwise comparisons. The fuzzy matrices of pairwise comparisons were formed for the criteria of both hierarchical levels. The data were the basis for the application of all the models considered: the FAHP, FBWM, and FUCOM-F. All three models were based on the principles of pairwise comparison of criteria and relations based on transitivity. Since all three models have similar basic mathematical foundations, it is possible to perform a comparison of results as well as testing based on the same data set. Thus, in this study, in order to compare the results of all 
three models, the data collected for the application of the FAHP model were used. For the pairwise comparison of all models, the same scale shown in Table 1 was used.

In order to form a FBWM mathematical model, fuzzy BO (Best-to-Others) and OW (Others-to-Worst) vectors must be formed. The fuzzy BO and OW vectors were formed on the basis of the comparisons made for the best/worst criterion in FAHP matrices. Similarly, the data from the AHP comparison matrices were used to form a mathematical model of the FUCOM-F. The comparisons were made for the most significant criterion and all criteria were ranked according to the data. Consequently, in Fig. 4 the final (global) values of the weight coefficients of the criteria applying the FAHP, FBWM, and FUCOM-F are shown.

In Fig. 4, it can be observed that the values of the weight coefficients of the criteria by the models considered are approximately the same if the central values of the interval of fuzzy numbers are taken into consideration. The greatest varieties of the fuzzy values of the weight coefficients of the criteria have been obtained with the FAHP model. For the majority of criteria, the weight values obtained using the FUCOM-F fit into the upper and lower limits of the fuzzy interval of the AHP model. It is similar to the FBWM. The values of weight coefficients using the FBWM also follow the AHP model intervals, except for the $C_{11}$ and $C_{13}$ criteria where the interval limits are shifted relative to the FAHP and the FUCOM-F. However, if we consider the maximum value belonging to fuzzy weight coefficient $C_{13}$, it is established that it is approximately the same as with the FAHP and the FUCOM-F. Thus, it can be concluded that, with respect to minor deviations, approximately the same values of weight coefficients have been attained from all of the models. In the following section, the comparison of the results was performed based on the deviations of the models considered from the maximum consistency.

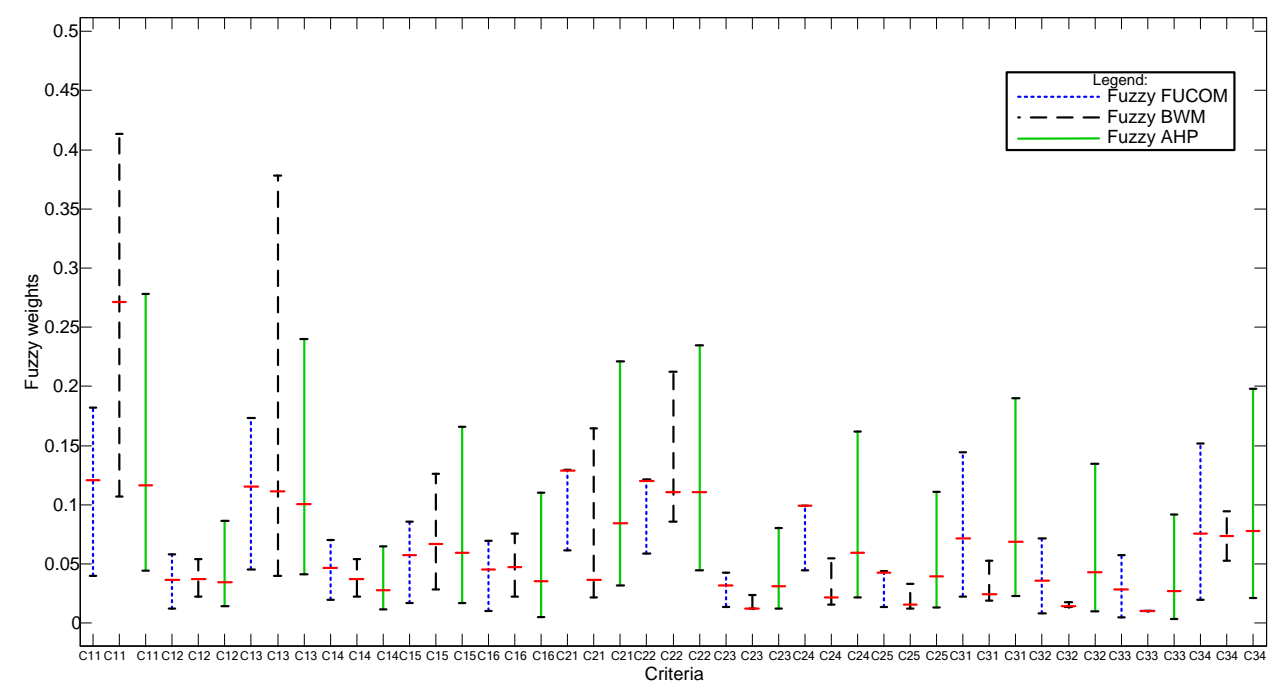

Fig. 4 Final values of the weights calculated by FAHP, FBWM, and FUCOM-F 


\subsection{Checking consistency}

The deviations were determined by hierarchical levels and the mean values of deviations by levels were identified. As with the FUCOM-F, one model was formed for the first level, while four models were formed for the second level. By means of the application of the FBWM, FAHP, and FUCOM-F, the following deviations from the maximum values of the degree of consistency with the models were obtained: (1) FBWM: $C R_{(\text {Model I })}=0.059, C R_{(\text {Model }}$ ${ }_{2)}=0.049, C R_{(\text {Model 3) }}=0.078$ and $C R_{(\text {Model 4) }}=0.074$; (2) FAHP: $C R_{(\text {Model I) }}=0.071, C R_{(\text {Model }}$ ${ }_{2)}=0.084, C R_{(\text {Model 3) }}=0.081$ and $C R_{(\text {Model 4) }}=0.080$; (3) FUCOM-F: $D F C_{(\text {Model 1) }}=0.001$, $D F C_{(\text {Model } 2)}=0.053, D F C_{(\text {Model I })}=0.074$ and $D F C_{(\text {Model } 4)}=0.066$.

Through analyzing the obtained deviations from the maximum consistency (DMC) for all three models, it can be perceived that the minimum consistency conditions defined by the FAHP model $\left(C R_{\min }=0.10\right)$ have been satisfied. The greatest deviations were obtained with the FAHP model, which was expected since it requires a greater number of comparisons of criteria $(n(n-1) / 2)$ compared to the FBWM $(2 n-3)$, and the FUCOM-F $(n-1)$. In addition, it has been perceived that the DMC values with the FUCOM-F and FBWM are approximately equal, with only slight differences. Yet, the FUCOM-F shows superior consistency with respect to the FBWM in three out of four models. However, it is necessary to emphasize that the deviations between these two models are minimal; so, greater dominance of the FUCOM-F over the FBWM cannot be discussed. Additionally, it is necessary to take into account the fact that there is a major difference in the number of the comparisons of criteria, especially regarding the relationship with the FAHP method. As a result, it can be anticipated that in certain cases, different results of the same problem, resolved by different methods, may be obtained. In the examples provided and comparisons with the FBWM and FAHP methods, there was no such case, but such a possibility should not be excluded from consideration.

\section{CONCLUSIONS}

When selecting the most suitable alternative for MCDM problems, different importance levels of the criteria are taken into consideration. In order to determine the importance levels with respect to the opinions of experts, a number of weighting methods such as the SAW, AHP/ANP, SWARA, BWM, and FUCOM have been used in existing literature. The Fuzzy set theory can be used to overcome problems containing ambiguity and vagueness. If such weighting methods are integrated with the fuzzy set theory, which best expresses the human thought and reasoning structure, more reliable results can be obtained. In this study, therefore, fuzzy sets were combined with the FUCOM method and the fuzzy FUCOM (FUCOM-F) has been proposed. Moreover, pairwise comparisons for criteria were conducted using linguistic variables instead of crisp values in the decisionmaking process.

One of the most important advantages of the proposed model is the provision of similar results as with the FBWM and the FAHP models by means of conducting solely $n$ 1 pairwise comparisons. Thereby, the influence of the inconsistency of expert preferences regarding the final values of the weights of criteria is reduced. Due to the minimum number of expert comparisons required, the FUCOM-F is considered to be the best way to determine the criteria weights. Furthermore, it is a simple mathematical apparatus that 
provides credible weight coefficients which contribute to rational judgment in decisionmaking [22]. As a result, the FUCOM-F is an effective decision-tool that aids decisionmakers in dealing with their own subjectivity when prioritizing criteria.

In the present paper, additionally, the robustness and objectivity of the proposed model has been demonstrated by comparing it with the FBWM and the FAHP models. The impressive consistency of the results obtained has been presented as well. Furthermore, it has been shown that the model is adjustable and suitable for application to various measuring scales in order to express expert preferences.

For future research, the proposed model could be applied in all areas of science, engineering, and social sciences. When combined with other ranking methods (TOPSIS, ARAS, EDAS, CODAS, MAIRCA, COPRAS, etc.), it could be utilized reliably in deciding on the best alternative for MCDM problems.

\section{REFERENCES}

1. Rezaei, J., 2015, Best-worst multi-criteria decision-making method, Omega, 53, pp. 49-57.

2. Vinogradova, I., Podvezko, V., Zavadskas, E., 2018, The recalculation of the weights of criteria in MCDM methods using the Bayes approach, Symmetry, 10(6), 205.

3. Roberts, R., Goodwin, P., 2002, Weight approximations in multi-attribute decision models, Journal of Multicriteria Decision Analysis, 11, pp. 291-303.

4. Cook, W.D., 2006, Distance-based and ad hoc consensus models in ordinal preference ranking, European Journal of Operational Research, 172, pp.369-385.

5. Zavadskas, E. K., Stević, Ž., Tanackov, I., \& Prentkovskis, O., 2018, A novel multicriteria approach-rough step-wise weight assessment ratio analysis method (R-SWARA) and its application in logistics, Studies in Informatics and Control, 27(1), pp. 97-106.

6. Zavadskas, E.K., Govindan, K., Antucheviciene, J., Turskis, Z., 2016, Hybrid multiple criteria decisionmaking methods: a review of applications for sustainability issues, Economic Research-Ekonomska Istraživanja, 29(1), pp. 857-887.

7. Milicevic, A., Pavlicic, D., Kostic, A., 2007, The dynamics of change in decision making under risk, Psihologija, 40(1), pp. 147-164.

8. Pamucar, D., Lukovac, V., Bozanic, D., Komazec, N., 2018b, Multi-criteria FUCOM-MAIRCA model for the evaluation of level crossings: case study in the Republic of Serbia, Operational Research in Engineering Sciences: Theory and Applications, 1(1), pp. 108-129.

9. Pamučar D, Stević, Ž., Sremac, S., 2018, A New Model for Determining Weight Coefficients of Criteria in MCDM Models: Full Consistency Method (FUCOM), Symmetry, 10(9), 393, pp. 1-22.

10. Bozanic, D., Tešić, D., Kočić, K., 2019, Multi-Criteria FUCOM - Fuzzy MABAC Model for the Selection of Location for Construction of Single-Span Bailey Bridge, Decision Making: Applications in Management and Engineering, 2 (1), pp. 132-46.

11. Bozanic, D., Tešić, D., Milić, A., 2020, Multicriteria Decision Making Model With Z-Numbers Based on FUCOM and MABAC Model, Decision Making: Applications in Management and Engineering 3(2), pp. 19-36.

12. Durmić, E., Stević, Ž., Prasenjit, C., Vasiljević, M., Tomašević, M., 2020, Sustainable Supplier Selection Using Combined FUCOM - Rough SAW Model, Reports in Mechanical Engineering, 1(1), pp. 34-43.

13. Badi, I., Abdulshahed, A., 2019, Ranking the Libyan airlines by using full consistency method (FUCOM) and analytical hierarchy process $(A H P)$, Operational Research in Engineering Sciences: Theory and Applications, 2(1), pp. 1-14.

14. Noureddine, M., Ristic, M., 2019, Route planning for hazardous materials transportation: Multicriteria decision making approach, Decision Making: Applications in Management and Engineering, 2(1), pp. 66-85.

15. Erceg, Z, \& Mularifovic, F., 2019, Integrated MCDM model for processes optimization in supply chain management in wood company, Operational Research in Engineering Sciences: Theory and Applications, 2(1), pp. 37-50.

16. Prentkovskis, O., Erceg, Z., Stevic, Z., Tanackov, I., Vasiljevic, M., Gavranovic, M. A., 2018, New Methodology for Improving Service Quality Measurement: Delphi-FUCOM-SERVQUAL Model, Symmetry, 10, pp. 757. 
Prioritizing the Weights of the Evaluation Criteria Under Fuzziness: the Fuzzy Full Consistency Method... 437

17. Zadeh, L.A., 1975, The concept of a linguistic variable and its application to approximate reasoning Part I, Information Sciences, 8(3), pp. 199-249.

18. Ecer, F., 2014, A hybrid banking websites quality evaluation model using AHP and COPRAS-G: a Turkey case, Technological and Economic Development of Economy, 20(4), pp. 758-782.

19. Ecer, F., 2018, An integrated Fuzzy AHP and ARAS model to evaluate mobile banking services, Technological and Economic Development of Economy, 24(2), pp. 670-695.

20. Guo, S., Zhao, H., 2017, Fuzzy best-worst multi-criteria decision-making method and its applications, Knowledge-Based Systems, 121, pp. 23-31.

21. Wang, B., Songa, J., Ren, J., Lib, K., Duana, H., Wanga, X., 2019, Selecting sustainable energy conversion technologies for agricultural residues: A fuzzy AHP-VIKOR based prioritization from life cycle perspective, Resources, Conservation \& Recycling, 142, pp. 78-87.

22. Fazlollahtabar, H., Smailbasic, A., Stevic, Z., 2019, FUCOM method in group decision-making: Selection of forklift in a warehouse, Decision Making: Applications in Management and Engineering, 2(1), pp. 49-65. 